INPLASY

PROTOCOL

To cite: Yao et al. Flavonoids on insulin resistance in overweight and obesity subjects: protocol for a systematic review and metaanalysis of randomized controlled trials. Inplasy protocol 202220011. doi: 10.37766/inplasy2022.2.0011

Received: 06 February 2022

Published: 06 February 2022

Corresponding author: Jia Yao

jiayaomomeak@163.com

Author Affiliation: Guangzhou University of Chinese Medicine.

Support: 2015BAI04B09.

Review Stage at time of this submission: Preliminary searches.

Conflicts of interest: None declared.

\section{Flavonoids on insulin resistance in overweight and obesity subjects: protocol for a systematic review and meta-analysis of randomized controlled trials}

\author{
Yao, J1; Zhao, J2; Yang, Z3; Lin, Y4; Lu, Q5; Xie, W6; Wen, J7; \\ Fan, $\mathrm{G}^{8}$.
}

Review question / Objective: Herbal and "natural" products are a growing industry in today's society because they reportedly help with numerous diseases and ailments. To date, there are some randomized controlled trials (RCTs) conducted on patients concerning the efficacy of flavonoids on insulin resistance in overweight and obesity patients that have shown inconsistent results. On this basis, we will summarize the available evidence to investigate the efficacy of flavonoids on on insulin resistance in overweight and obesity patients by conducting a systematic review and metaanalysis.

Condition being studied: To date, there are some RCTs conducted on patients concerning the efficacy of flavonoids on insulin resistance in overweight and obesity patients. However, the RCTs have shown inconsistent results and the evidence of the effects of flavonoids on insulin resistance in overweight and obesity patients remains decentralized.

INPLASY registration number: This protocol was registered with the International Platform of Registered Systematic Review and Meta-Analysis Protocols (INPLASY) on 06 February 2022 and was last updated on 06 February 2022 (registration number INPLASY202220011).

\section{INTRODUCTION}

Review question / Objective: Herbal and "natural" products are a growing industry in today's society because they reportedly help with numerous diseases and ailments. To date, there are some randomized controlled trials (RCTs) conducted on patients concerning the efficacy of flavonoids on insulin resistance in overweight and obesity patients that have shown inconsistent results. On this basis, we will summarize the available evidence to investigate the efficacy of flavonoids on on insulin resistance in overweight and 
obesity patients by conducting a systematic review and meta-analysis.

Condition being studied: To date, there are some RCTs conducted on patients concerning the efficacy of flavonoids on insulin resistance in overweight and obesity patients. However, the RCTs have shown inconsistent results and the evidence of the effects of flavonoids on insulin resistance in overweight and obesity patients remains decentralized.

\section{METHODS}

Search strategy: PubMed \#1 Search "flavonoid" [MeSH Terms] \#2 Search "insulin resistance" [MeSH Terms] \#3 Search (flavonoid* OR flavonol OR flavone OR flavanone OR flavan-3-ol OR anthocyanidin OR isoflavone OR quercetin OR kaempferol OR myricetin OR isorhamnetin OR luteolin OR apigenin OR hesperetin OR naringenin OR catechin OR epicatechin OR epigallocatechin OR e p i c a tech in-3-ga I l a te OR epigallocatechin-3-gallate OR cyanidin OR delphinidin OR malvidin OR pelargonidin OR petunidin OR peonidin OR genistein OR daidzein OR hespertin OR proanthocyanidin): Title/Abstract/Text Word \#4 Search ("insulin resistance" OR "insulin sensitivity" OR "hyperinsulinemic euglycemia clamp" OR "euglycemic clamp" OR "glucose clamp" OR "glucose infusion rate" OR GIR OR "oral glucose tolerance test" OR OGTT OR "insulin tolerance test" OR ITT OR "homeostasis model assessment" OR HOMA OR HOMA-IAI OR HOMA-IR OR HOMA-IS OR HOMA- $\beta$ OR "quantitative insulin sensitivity check index" OR QUICKI OR "fasting insulin resistance index" OR FIRI OR "insulinogenic index" OR IGI OR "minimal model analysis" OR "minimal model" OR "index of insulin sensitivity" OR "insulin sensitivity index" OR ISI OR "fasting insulin" OR FINS OR "fasting glucose" OR FPG): Title/Abstract/Text Word \#5 Search ('randomized controlled trial' OR RCT OR randomized OR clinical OR trial OR randomly): All Fields

\#6 Search \#1 OR \#3

\#7 Search \#2 OR \#4
\#8 Search \#6 AND \#7 AND \#5.

Participant or population: Overweight or obesity patients.

Intervention: Flavonoids.

Comparator: The control group.

Study designs to be included: RCTs.

Eligibility criteria: Study design: RCTs published with any follow-up duration and sample size will be included in this systematic review and meta-analysis. Participants: patients who suffered from overweight or obesity. There will be no restrictions for gender, age, or ethnicity.InterventionsThe interventions for treating overweight and obesity in the experimental group could be any kinds of flavonoids (including flavonols, flavones, flavanones, flavan-3-ols, anthocyanidins, isoflavones, quercetins, kaempferols, myricetins, isorhamnetins, luteolins, apigenins, hesperetins, naringenins, catechins, epicatechins, epigallocatechins, epicatechin-3-gallates, epigallocatechin-3gallates, cyanidins, delphinidins, malvidins, pelargonidins, petunidins, peonidins, genisteins, daidzeins, hespertins, and proanthocyanidins).

Information sources: The Pubmed, Embase, Web of Science, and the Cochrane Central Register of Controlled Trials will be searched from inception time to date. The ClinicalTrials.gov registry will also be searched for unpublished trials and the authors were contacted for additional information if necessary. Relevant references from included studies were sought to retrieve additional eligible studies. No limits were set on language, publication year, and type of publication.

Main outcome(s): The primary outcomes: (1) HOMA-IR; (2) QUCIKI; (3) ISI.

Additional outcome(s): The secondary outcomes: (1) Fasting blood glucose; (2) Fasting insulin; (3) Blood lipids: total cholesterol, triglyceride, HDL-C, LDL-C; (4) Blood pressure: SBP, DBP; (5) Body weight; 
(6) BMI; (7) Waist circumference; (8) Waistto-hip ratio; (9) incidence of adverse reactions.

Data management: For each eligible trial, pairs of reviewers, following training and calibration exercises, extracted data independently by using a standardized, pilot-tested data extraction form. Reviewers collected information on trial characteristics, patient characteristics, experimental and control interventions, duration, and outcomes of interest. Reviewers resolved discrepancies by discussion and, when necessary, with adjudication by a third party. When relevant details were insufficiently reported in studies, authors will be contacted by email, and the ClinicalTrials.gov register was searched for further information.

Quality assessment / Risk of bias analysis: For each eligible trial, reviewers, following training and calibration exercises, will use a revision of the Cochrane tool for assessing the risk of bias in randomized trials (RoB 2.0) to rate trials as either at (1) low risk of bias, (2) some concerns-probably low risk of bias, (3) some concerns-probably high risk of bias, or (4) high risk of bias, across the following domains: randomization process, deviations from intended interventions, missing outcome data, measurement of the outcome, and selection of the reported result. Within each domain, the assessment comprised a series of signaling questions; a judgment about risk of bias for the domain, facilitated by an algorithm that maps responses to signaling questions to a proposed judgment; free text boxes to justify responses to the signaling questions and risk-of-bias judgments; and optional free text boxes to predict (and explain) the likely direction of bias. Furthermore, we assessed the risk of bias of included RCTs by using version 2 of the Cochrane risk-ofbias assessment tool for randomized trials. We rated trials at low risk of bias overall if the study was judged to be at low risk of bias for all domains for this result; rated trials at some concerns of bias overall if the study was judged to be at some concerns in at least one domain for this result, but not to be at high risk of bias for any domain; and rated trials at high risk of bias overall if the study was judged to be at high risk of bias in at least one domain for this result or was judged to have some concerns for multiple domains in a way that substantially lowers confidence in the result. Reviewers resolved discrepancies by discussion and, when not possible, with adjudication by a third party.

Strategy of data synthesis: Stata, version 16.0 (StataCorp LLC) was used for statistical analysis. Continuous data used the weighted mean difference (WMD) with 95\% confidence intervals (Cl) after the units are standardized. Dichotomous data used the relative risk (RR) with $95 \% \mathrm{CI}$. Missing data were dealt with according to the Cochrane Handbook for Systematic Reviews of Interventions. $P<0.05$ was considered statistically significant. Heterogeneity was tested by $\mathrm{X} 2$-based Cochran $Q$ statistic $(P<0.10$ indicated statistically significant heterogeneity) and I2 statistic. If $12<50 \%$, a fixed-effects model was used to pool the estimations across studies. If $12 \geq 50 \%$, after excluding clinical heterogeneity between studies, the random-effects model was used. Quantitative data, where possible, were pooled for meta-analysis. Where pooling was not possible, the findings were presented in a narrative form. Publication bias was examined according to the funnel plot method. The Egger's test and Begg's test were conducted to quantitatively assess the publication bias, furthermore, the trim and fill method was used to correct the funnel asymmetry caused by publication bias.

Subgroup analysis: Types of intervention drugs.

Sensitivity analysis: Sensitivity analysis was conducted by excluding studies one by one so that we can determine the source of heterogeneity.

Country(ies) involved: China.

Keywords: Flavonoids; insulin resistance; overweight; obesity; meta-analysis. 
Contributions of each author:

Author 1 - Jia Yao.

Email: jiayaomomeak@163.com

Author 2 - Jia Zhao.

Author 3 - Zhaojun Yang.

Author 4 - Yuping Lin.

Author 5 - Qiyun Lu.

Author 6 - Wenwen Xie.

Author 7 - Jianxuan Wen.

Author 8 - Guanjie Fan.

Email: fanguanjiegz@163.com 